\title{
MODULAR GROUP ALGEBRAS OF SIMPLY PRESENTED ABELIAN GROUPS
}

\author{
WARREN MAY
}

(Communicated by Bhama Srinivasan)

\begin{abstract}
Let $G$ be a $p$-local Warfield group and $F$ a perfect field of characteristic $p$. It is shown that if the group algebras $F G$ and $F H$ are isomorphic, then $G$ and $H$ are isomorphic.
\end{abstract}

1. Introduction. Let $p$ be a prime number. Throughout this paper $F$ will denote a perfect field of characteristic $p$, and $G$ will denote an abelian group which will be written multiplicatively.

If $H$ is a group such that the group algebras $F G$ and $F H$ are $F$-isomorphic, then it has been known for some time that the Ulm $p$-invariants of $G$ and $H$ must be equal $[\mathbf{2}, \mathbf{5}, \mathbf{9}]$. Consequently, $G$ and $H$ are isomorphic if they are simply presented abelian $p$-groups. We remark that the Ulm invariant at $p$-height $\infty$ is included above, thus $G$ and $H$ need not be reduced. Suppose, however, one hypothesizes that only $G$ is a simply presented $p$-group. The question then arises whether $H$ must be one also. This question has resisted resolution except in special cases. If $G$ is countable, then $H$ is necessarily simply presented. If $G$ is a direct sum of cyclic $p$-groups, then Berman and Mollov [3] proved that $H$ is one also. It was then shown in [10] that if $G$ is simply presented of $p$-length less than the first uncountable ordinal, then the same is true of $H$. This restriction on length was due to the use of a theorem of P. Hill in which such a limitation was necessary.

In this paper, we shall settle the question for simply presented $p$-groups by using a more direct approach. We let $V(G)$ denote the group of normalized units (i.e. of augmentation 1) in $F G$. Our results will be consequences of

THEOREM 1. $V(G)$ is a simply presented abelian p-group if and only if $G$ is a simply presented abelian p-group.

The Ulm invariants of $V(G)$ have been computed for $F$ a perfect field, therefore the structure of $V(G)$ is determined for $G$ simply presented.

Before stating an isomorphism theorem, we give an immediate application of Theorem 1 to the direct factor problem. This problem asks whether $G$ is a direct factor of $V(G)$. Discussions of this question can be found in [4 and 11]. For simply presented $p$-groups, the result on splitting proved in $[\mathbf{1}$, Theorem 5.2] is subsumed by

THEOREM (DIRECT FACTOR). If $G$ is a simply presented abelian p-group, then $G$ is a direct factor of $V(G)$.

Received by the editors March 1, 1987 and, in revised form, June 9, 1987, and November 20, 1987.

1980 Mathematics Subject Classification (1985 Revision). Primary 20K21; Secondary $20 \mathrm{C07.}$ 
We now give an isomorphism theorem in a more general setting than simply presented $p$-groups. The $p$-local Warfield groups are groups that may be mixed, and which are characterized as summands of simply presented modules over the ring of $p$-adic rational numbers. A reference for $p$-local Warfield groups is [7].

THEOREM 3 (ISOMORPHISM). If $G$ is a p-local Warfield group and $H$ is a group such that $F G \cong F H$, then $G \cong H$.

COROLLARY. If $G$ is a simply presented abelian p-group and $H$ is a group such that $F G \cong F H$, then $G \cong H$.

We remark that it was shown in $[\mathbf{1}]$ that this theorem holds if $H$ is also assumed to be a $p$-local Warfield group.

Two questions which these results raise are the following. Does the direct factor theorem hold for $p$-local Warfield groups $G$ ? This could easily be proved provided $V(G)$ is a Warfield group if $G$ is one; however, this is probably not true. To state the second question, let $t G$ denote the torsion subgroup of an abelian group $G$. If $t G$ is a simply presented $p$-group, and if $F G \cong F H$, then is $t G \cong t H$ ?

Finally, we wish to point out the importance of the isomorphism problem for groups with only $p$-torsion over characteristic $p$ fields. It is shown by Ullery in [14] that resolution of this problem is central to that for abelian group algebras over arbitrary coefficient rings. Unfortunately, the likelihood of settling these matters in full generality seems quite remote.

The author is grateful to the referee for several corrections and clarifications.

2. Characteristic $p$ lemmas. As before, $F$ will denote a perfect field of characteristic $p$. If $N \leq G$, then the natural map $F G \rightarrow F(G / N)$ of group algebras induces a homomorphism $V(G) \rightarrow V(G / N)$ whose kernel we denote by $K(N)$. Letting $I(N)$ denote the augmentation ideal of $F N$, we can write $K(N)=V(G) \cap$ $(1+F G \cdot I(N))$.

LEMMA 1. If $t G$ denotes the torsion subgroup of $G$, then $V(G)=G \cdot K(t G)$ and $G \cap K(t G)=t G$.

Proof. Since $G / t G$ is torsion-free, it is well known that $V(G / t G)=G / t G$, hence the image of $G$ under the natural map $V(G) \rightarrow V(G / t G)$ is the whole image. Thus $V(G)=G \cdot K(t G)$. The second statement is obvious.

LEMMA 2. (1) Let $N \leq G, N$ a p-group. Under the natural map $F G \rightarrow$ $F(G / N)$, any preimage of an element of $V(G / N)$ lies in $V(G)$. The map $V(G) \rightarrow$ $V(G / N)$ is surjective, and $K(N)=1+F G \cdot I(N)$ is a p-group.

(2) If $G$ is a p-group, then $V(G)=1+I(G)$ is a p-group.

(3) If $t G$ is a p-group, then $V(G) / G$ is a p-group.

Proof. The kernel of $F G \rightarrow F(G / N)$ is $F(G) \cdot I(N)$, which is a nil ideal since $\left(\sum a_{i} g_{i}\right)^{p^{n}}=0$ if $a_{i} \in F, g_{i} \in N, \sum a_{i}=0$ and $g_{i}^{p^{n}}=1(1 \leq i \leq k)$. Thus any preimage of a unit is a unit. The rest of (1) now follows easily. Clearly, (1) implies (2) since $K(G)=V(G)$. In the situation of (3), (1) implies that $K(t G)$ is a $p$-group, thus Lemma 1 proves (3).

We remark on a representation for elements of $K(N)$ that we will use later. Under the assumption of (1) above, suppose that $\left\{g_{i}: i<\nu\right\}$ is a family of coset 
representatives of $N$ in $G$ for some ordinal $\nu$. Then the elements of $K(N)$ are precisely the sums $1+\sum_{i<\nu} g_{i} \alpha_{i}, \alpha_{i} \in I(N)$ with almost all $\alpha_{i}=0$. Moreover, the $\alpha_{i}$ in this representation are unique.

Next we wish to discuss $p$-heights for abelian groups and commutative rings of characteristic $p$. We shall refer to these as heights since $p$ is fixed. If $S$ is either a group or ring as above, define $S^{\sigma}$ for every ordinal $\sigma$ in the usual manner: $S^{0}=S$; $S^{\sigma+1}=\left(S^{\sigma}\right)^{p}$; and $S^{\sigma}=\bigcap_{\tau<\sigma} S^{\tau}$ if $\sigma$ is a limit ordinal. If $\sigma$ is the first ordinal such that $S^{\sigma}=S^{\sigma+1}$, we call $\sigma$ the $p$-length of $S$ and define $S^{\infty}$ to be $S^{\sigma}$. Then $S^{\infty}$ is the maximal $p$-divisible subgroup or subring of $S$. If $s \in S$, we define the height $h(s)$ to be $\infty$ if $s \in S^{\infty}$, and the smallest ordinal $\sigma$ such that $s \notin S^{\sigma+1}$ otherwise. Note that $F^{\sigma}=F$ for every $\sigma$ since $F$ is perfect. Since $\left(\sum a_{i} g_{i}\right)^{p}=\sum a_{i}^{p} g_{i}^{p}$, it is easily shown that $(F G)^{\sigma}=F G^{\sigma}$ and $V(G)^{\sigma}=V(G) \cap F G^{\sigma}$. Thus $h\left(\sum a_{i} g_{i}\right)=\min \left\{h\left(g_{i}\right)\right\}$, and the notions of height in $G, V(G)$ and $F G$ agree whenever a comparison can be made.

LEMMA 3. Let $A$ be a subgroup of $G$. The following are equivalent:

(1) $A$ is isotype in $G$;

(2) $A$ is isotype in $V(G)$;

(3) $V(A)$ is isotype in $V(G)$.

ProOF. The equivalence of (1) and (2) follow from the remark above on heights in $G$ and $V(G)$. To show that (1) and (3) are equivalent, we first note that if $B \leq G$, then $V(A) \cap F B=V(A \cap B)$ (see [8, p. 37]). Thus $V(A) \cap V(G)^{\sigma}=V(A) \cap F G^{\sigma}=$ $V\left(A \cap G^{\sigma}\right)$, and $V(A)^{\sigma}=V(A) \cap F A^{\sigma}=V\left(A^{\sigma}\right)$. Equality will hold precisely when $A^{\sigma}=A \cap G^{\sigma}$.

LEMMA 4. (1) Let $N \leq G, N$ a p-group. Then $N$ is nice in $G$ if and only if $K(N)$ is nice in $V(G)$.

(2) Let $N \leq G$. Then $N$ is nice in $G$ if and only if $N$ is nice in $V(G)$.

Proof. (1) By Lemma 2, $V(G) / K(N)=V(G / N)$. Assume that $N$ is nice in $G$ and that $\sum a_{i}\left(g_{i} N\right) \in V(G / N)$. We may suppose the $g_{i}$ are chosen such that $h\left(g_{i}\right)=h\left(g_{i} N\right)$. (It should be clear from context in what groups heights are computed.) By Lemma 2, $\sum a_{i} g_{i} \in V(G)$ and $h\left(\sum a_{i} g_{i}\right)=h\left(\sum a_{i}\left(g_{i} N\right)\right)$. Thus $K(N)$ is nice in $V(G)$.

For the converse, let $g \in G$. Since $K(N)$ is nice in $V(G)$, we may choose $\alpha \in K(N)$ such that $h(g \alpha)=h(g N)$. By examining the representation of $\alpha$ given after Lemma 2 , the support of $\alpha$ must contain an element $n \in N$. But $h(g n) \geq$ $h(g \alpha)=h(g N)$, thus $h(g n)=h(g N)$ and $N$ is nice in $G$.

(2) By Lemma 3, $G$ is isotype in $V(G)$. Therefore it suffices to show that $G$ is nice in $V(G)$. Let $\alpha \in V(G)$ and consider $G \alpha$. Altering $\alpha$ by a factor from $G$, we may assume that $e$ lies in the support of $\alpha$. Suppose that $g \in G$ with $h(g)=h(\alpha)$. Then clearly $h(g \alpha) \leq h(g)=h(\alpha)$, thus $G$ is nice in $V(G)$.

3. Simply presented $G$. Let $A \leq B \leq G$. By a smooth chain from $A$ to $B$ we mean a family of subgroups $\left\{N_{\sigma}: \sigma \leq \lambda\right\}$ for some ordinal $\lambda$, such that $N_{0}=A$, $N_{\lambda}=B, N_{\tau} \subseteq N_{\sigma}$ if $\tau<\sigma$, and $N_{\sigma}=\bigcup_{\tau<\sigma} N_{\tau}$ if $\sigma$ is a limit ordinal. If, in addition, $\left(N_{\sigma+1}: N_{\sigma}\right)=p$ for every $\sigma<\lambda$, we say we have a composition series from $A$ to $B$. We call it a nice composition series if every $N_{\sigma}$ is nice in $G$. Then $G$ is simply presented if there exists a nice composition series from 1 to $G$. 
Now let $S \leq G, S$ a $p$-group. We want to define certain subgroups of $K(S)$ in order to construct a nice composition series. Let $M=I(S)$ be the augmentation ideal of $F S$, and choose a family $\left\{g_{i}: i<\nu\right\}$ of coset representatives of $S$ in $G$. By the remark after Lemma 2, each $\alpha \in K(S)$ has a unique representation $\alpha=1+\sum_{i<\nu} g_{i} \alpha_{i}$, where $\alpha_{i} \in M$. Note that $h(\alpha)=\min \left\{h\left(g_{i} \alpha_{i}\right): i<\nu\right\}$ since the supports of the $g_{i} \alpha_{i}$ are disjoint. Let $k \geq 1, \sigma \leq \nu$. We define

$$
N_{k, \sigma}=1+\sum_{i<\sigma} g_{i} M^{k}+\sum_{\sigma \leq i<\nu} g_{i} M^{k+1}
$$

where $M^{k}$ is the ideal product in $F S$. It is easy to see that $N_{k, \sigma}$ is closed under multiplication since $g_{i} M^{a} g_{j} M^{b} \subseteq g_{m} M^{k+1}$ if $a, b \in\{k, k+1\}$ and $g_{i} g_{j} S=g_{m} S$. Since $K(S)$ is a $p$-group by Lemma $2, N_{k, \sigma}$ is a subgroup of $K(S)$. If $1 \leq k \leq r$, then $\left\{N_{k, \sigma}: 1 \leq k \leq r, \sigma \leq \nu\right\}$ is a smooth chain from $N_{r, 0}$ to $N_{1, \nu}=K(S)$. The chain is ordered by the lexicographic order on $(-k, \sigma)$.

LEMMA 5. Let $S \leq G, S$ a finite p-group. If $N_{k, \sigma} \leq N \leq N_{k, \sigma+1}$, then every coset of $N$ in $N_{k, \sigma+1}$ has an element proper in $V(G)$ with respect to $N$.

ProOF. Fix $\alpha \in N_{k, \sigma+1}$. Let $\beta \in N$ and write $\alpha \beta=1+\sum_{i<\nu} g_{i} \alpha_{i}\left(\alpha_{i} \in M^{k}\right)$ in the unique representation above. Note for later use that $h(\alpha \beta) \leq h\left(g_{\sigma} \alpha_{\sigma}\right)$. Put $\beta^{\prime}=\alpha^{-1}\left(1+g_{\sigma} \alpha_{\sigma}\right)$. Then $\beta^{\prime} \in N_{k, \sigma+1}$ since $1+g_{\sigma} \alpha_{\sigma} \in N_{k, \sigma+1}$. Putting $\gamma=\sum_{i<\nu} g_{i} \alpha_{i}$, we have $\alpha \beta=1+\gamma$, where $\gamma$ is nilpotent. Thus $(\alpha \beta)^{-1}=$ $1-\gamma+\gamma^{2}-\cdots=1-\gamma+\delta$, where $\delta \in \sum_{i<\nu} g_{i} M^{k+1}$. Consequently $\beta^{-1} \beta^{\prime}=$ $(1-\gamma+\delta)\left(1+g_{\sigma} \alpha_{\sigma}\right)=1-\gamma+g_{\sigma} \alpha_{\sigma}+\delta^{\prime}$, where $\sigma^{\prime} \in \sum_{i<\nu} g_{i} M^{k+1}$. The $g_{\sigma}$ term of $1-\gamma$ is $-g_{\sigma} \alpha_{\sigma}$, hence $\beta^{-1} \beta^{\prime} \in N_{k, \sigma} \subseteq N$. Therefore $\beta^{\prime} \in N$. Moreover, $\alpha \beta^{\prime}=$ $1+g_{\sigma} \alpha_{\sigma}$, thus $h\left(\alpha \beta^{\prime}\right)=h\left(g_{\sigma} \alpha_{\sigma}\right) \geq h(\alpha \beta)$. Now we note that $\alpha_{\sigma}$ depends on $\beta$ by writing it as $\alpha_{\sigma}(\beta)$. Thus we see that $\sup \{h(\alpha \beta): \beta \in N\}=\sup \left\{h\left(g_{\sigma} \alpha_{\sigma}(\beta)\right): \beta \in\right.$ $N\}$. But $g_{\sigma} \alpha_{\sigma}(\beta)$ has support in $g_{\sigma} S$, which is a finite set. Hence the supremum is achieved.

For $S$ finite as above, $F S$ is noetherian and hence $M$ is nilpotent, say $M^{r+1}=0$. Then $N_{r, 0}=1$ and $\left\{N_{k, \sigma}: 1 \leq k \leq r, \sigma \leq \nu\right\}$ is a smooth chain from 1 to $K(S)$. We may interpolate additional subgroups to obtain a composition series from 1 to $K(S)$. By the lemma, each subgroup is nice in the next, where heights are computed in $V(G)$. Under these circumstances, the relation "is nice in" is transitive, hence it follows that each subgroup is nice in $V(G)$ since $K(S)$ is nice by Lemma 4 . Thus we have the

COROLlaRY. Let $S \leq G, S$ a finite p-group. Then there is a nice composition series from 1 to $K(S)$ in $V(G)$.

PROPOSITION 6. If $G$ is a simply presented p-group, then $V(G)$ is a simply presented p-group.

ProOF. Let $\left\{N_{\sigma}: \sigma \leq \lambda\right\}$ be a nice composition series for $G$. By Lemma 4, $\left\{K\left(N_{\sigma}\right): \sigma \leq \lambda\right\}$ is a chain of nice subgroups of $V(G)$ from 1 to $V(G)$. Since an element of $V(G)$ has finite support, it is clear that it is a smooth chain. By Lemma 2, $V(G) / K\left(N_{\sigma}\right)=V\left(G / N_{\sigma}\right)$, and clearly $K\left(N_{\sigma+1}\right) / K\left(N_{\sigma}\right)=K\left(N_{\sigma+1} / N_{\sigma}\right)$. By the corollary above, there is a nice composition series from 1 to $K\left(N_{\sigma+1} / N_{\sigma}\right)$, hence there is one from $K\left(N_{\sigma}\right)$ to $K\left(N_{\sigma+1}\right)$. We thus obtain a nice composition series from 1 to $V(G)$, as desired. 
4. Simply presented $V(G)$. Let $X \subseteq V(G)$ be a subset. We define the support $[X]$ of $X$ to be the union of supports of the elements of $X$. We call $X$ saturated if $[X] \subseteq X$. Thus $X$ is saturated if and only if $X \cap G=[X]$. The usefulness of saturated subgroups is revealed by

LEMMA 7. Let $N \leq V(G)$ be saturated. If $N$ is nice in $V(G)$, then $N \cap G$ is nice in $G$.

Proof. Let $g \in G$. Then there exists $\alpha \in N$ such that $h(g \alpha)$ is maximum in $g N$. Choose $g_{1}$ in the support of $\alpha$ such that $h(g \alpha)=h\left(g g_{1}\right)$. Then $g_{1} \in N \cap G$, and if $g_{2} \in N \cap G$, we have $h\left(g g_{2}\right) \leq h(g \alpha)=h\left(g g_{1}\right)$. Therefore $N \cap G$ is nice in $G$.

Recall that a $p$-group is simply presented if and only if it has a nice system $\Gamma$. That is, $\Gamma$ is a set of nice subgroups that contains the trivial subgroup, arbitrary products of members of $\Gamma$, and if $N \in \Gamma$ and $X$ is a countable subset of the group, then there exists $N^{\prime} \in \Gamma$ such that $N^{\prime} \supseteq N \cup X$ and $\left(N^{\prime}: N\right) \leq \aleph_{0}$.

LEMMA 8. Let $V(G)$ have a nice system $\Gamma$. Suppose that $N \in \Gamma$ is saturated and that $X \subseteq V(G)$ is a countable subset. Then there exists $N^{\prime} \in \Gamma$ such that $N^{\prime}$ is saturated, $N^{\prime} \supseteq N \cup X$, and $\left(N^{\prime}: N\right) \leq \aleph_{0}$.

Proof. Put $N_{0}=N$. Choose $N_{1} \in \Gamma$ such that $N_{1} \supseteq N_{0} \cup X$ and $\left(N_{1}: N_{0}\right) \leq$ $\aleph_{0}$. Note that $\left[N_{0}\right] \subseteq N_{1}$. Assume by induction that we have $N_{0} \subseteq \cdots \subseteq N_{k}$ such that $N_{i} \in \Gamma,\left(N_{i}: N_{0}\right) \leq \aleph_{0}$ and $\left[N_{i-1}\right] \subseteq N_{i}(1 \leq i \leq k)$. Then $N_{k}=\left\langle N_{k-1}, Y\right\rangle$ for some countable set $Y \subseteq V(G)$. There exists $N_{k+1} \in \Gamma$ such that $N_{k+1} \supseteq$ $N_{k} \cup[Y]$ and $\left(N_{k+1}: N_{k}\right) \leq \aleph_{0}$. But $\left[N_{k}\right] \subseteq\left[N_{k-1}\right] \cdot[Y] \subseteq N_{k+1}$, completing the induction. Put $N^{\prime}=\bigcup_{i<\omega} N_{i}$. Then $N^{\prime} \in \Gamma, N^{\prime} \supseteq N \cup X,\left(N^{\prime}: N\right) \leq \aleph_{0}$ and $\left[N^{\prime}\right]=\bigcup_{i<\omega}\left[N_{i}\right] \subseteq N^{\prime}$.

PrOpOSITION 9. If $V(G)$ is a simply presented p-group, then $G$ is a simply presented $p$-group.

Proof. Choose a nice system $\Gamma$ for $V(G)$ and put $\Gamma_{0}=\{N \cap G: N \in \Gamma, N$ saturated $\}$. It suffices to show that $\Gamma_{0}$ is a nice system for $G$. In view of Lemmas 7 and 8 , we only need show that $\Gamma_{0}$ is closed under arbitrary products. Therefore, suppose that $N_{i} \in \Gamma, N_{i}$ saturated $(i \in I)$, and consider $\coprod_{i \in I}\left(N_{i} \cap G\right)$. Then $\left.\amalg\left(N_{i} \cap G\right) \subseteq\left(\amalg N_{i}\right) \cap G \subseteq \amalg N_{i}\right] \subseteq \amalg\left[N_{i}\right]=\amalg\left(N_{i} \cap G\right)$. Thus, equality throughout implies that $\amalg N_{i} \in \Gamma$ is saturated and that $\amalg\left(N_{i} \cap G\right)=\left(\amalg N_{i}\right) \cap G \in \Gamma_{0}$.

5. Proofs of the theorems. Theorem 1 follows immediately from Propositions 6 and 9. Thus, when $G$ is simply presented one may describe the structure of $V(G)$ by calculating its Ulm invariants. This has been done for $F$ a field by Mollov [12]. We now briefly describe these results when $F$ is a perfect field of characteristic $p$. Let $f(\sigma)$ denote the $\sigma$ th Ulm invariant of $V(G)$ and let $\lambda$ denote the $p$-length of $G$.

(1) If $G^{\infty}=1$, then $f(\infty)=0$; if $G^{\infty} \neq 1$, then $f(\infty)=|F|\left|G^{\infty}\right|$.

(2) If $\lambda \leq \sigma<\infty$, then $f(\sigma)=0$.

(3) If $\sigma<\lambda$ and either $|F|$ or $\left|G^{\sigma}\right|$ is finite, then $f(\sigma)=|F|\left|G^{\sigma}\right|$.

(4) If $\sigma<\lambda$ and both $|F|$ and $\left|G^{\sigma}\right|$ are finite, then

$$
f(\sigma)=\left(\left|G^{\sigma}\right|-2\left|G^{\sigma+1}\right|+\left|G^{\sigma+2}\right|\right) \log _{p}|F| .
$$

Theorem 2 will follow from Theorem 1 by applying a simple lemma and trick used in [10]. The lemma [10, Lemma 2] asserts that $G$ is a direct factor of $V(G)$ if $G$ is 
isomorphic to a direct factor of $V\left(G^{\prime}\right)$ for some group $G^{\prime}$. Take $G^{\prime}=\bigsqcup_{i<\omega} G$. Then every nonzero Ulm invariant of $G$ is multiplied by $\aleph_{0}$, and Lemma 3 implies that the Ulm invariants of $G^{\prime}$ do not exceed those of $V\left(G^{\prime}\right)$. Consequently, $G \times V\left(G^{\prime}\right)$ and $V\left(G^{\prime}\right)$ have the same Ulm invariants and are simply presented by Theorem 1 . The lemma referred to now finishes the proof of Theorem 2 .

The proof of Theorem 3 will require more work. We shall assume that $G$ is a $p$-local Warfield group and that $H$ is a group with $F G \cong F H$. It is well known that such an isomorphism may be assumed to preserve augmentation. We therefore write $F G=F H$ and think of $H$ as a subgroup of $V=V(G)=V(H)$ that is a linear basis for $F G$. A number of conclusions about $H$ now follow from Lemmas 1 and 2. Since $t G$ is a $p$-group, we see that $t H$ is a $p$-group. Now let $q \neq p$ be a prime. Since $G$ is $q$-divisible, the same follows for $V$. But $H / t H \cong V / K(t H)$, thus $H / t H$ is $q$-divisible, and hence so is $H$. Therefore $H$ is a $p$-local group which we proceed to show is a Warfield group.

Let $X^{\prime}$ be a nice decomposition basis of $G$ (see [7]). Since $V / H$ is torsion by Lemma 2, there exists a subordinate basis $X$ contained in $H$. But subordinates of nice decomposition bases remain nice decomposition bases. By Lemma 4, since $G$ and $H$ are isotype in $V$ with torsion quotients, we conclude that $X$ is a nice decomposition basis of $H$ as well as $G$. Therefore $G$ and $H$ have the same Warfield invariants, and, as remarked in the introduction, they are known to have the same Ulm invariants. Thus we will be done if we finish showing that $H$ is a Warfield group. Let $B=\langle X\rangle$. Then $F(G / B)=F G / F G \cdot I(B)=F H / F H \cdot I(B)=F(H / B)$. But $G$ a Warfield group implies that $G / B$ is a simply presented $p$-group. Theorem 1 implies $H\rfloor B$ is simply presented, therefore $H$ is a Warfield group.

\section{REFERENCES}

1. D. Beers, F. Richman and E. Walker, Group algebras of abelian groups, Rend. Sem. Mat. Univ. Padova 69 (1983), 41-46.

2. S. D. Berman, Group algebras of countable abelian p-groups, Publ. Math. Debrecen 14 (1967), 365-405.

3. S. D. Berman and T. Zh. Mollov, The group rings of abelian p-groups of arbitrary power (Russian), Mat. Zametki 6 (1969), 381-392; Math. Notes 6 (1969), 686-692.

4. R. K. Dennis, Units in group rings, J. Algebra 43 (1976), 655-664.

5. P. F. Dubois and S. K. Sehgal, Another proof of the invariance of Ulm's functions in commutative modular group rings, Math. J. Okayama Univ. 15 (1972), 137-139.

6. L. Fuchs, Infinite abelian groups, vol. II, Academic Press, New York, 1973.

7. R. Hunter, F. Richman and E. Walker, Warfield modules, Abelian Group Theory, Proc. New Mexico State Univ. Conf. (1976), Lecture Notes in Math., vol. 616, Springer-Verlag, New York, 1977, pp. 87-123.

8. G. Karpilovsky, Commutative group algebras, Dekker, New York, 1983.

9. W. May, Commutative group algebras, Trans. Amer. Math. Soc. 136 (1969), 139-149.

10. (1979), 31-34.

11. __ Unit groups and isomorphism theorems for commutative group algebras, Group and Semigroup Rings, Proc. Univ. Witwatersrand Conf. (1985), North-Holland Math. Studies, No. 126, North-Holland, Amsterdam, 1986, pp. 163-178. 
12. T. Zh. Mollov, Ulm invariants of Sylow p-subgroups of group algebras of abelian groups over fields of characteristic $p$, Pliska 2 (1981), 77-82.

13. T. Zh. Mollov and N. A. Nachev, Ulm-Kaplansky invariants of the group of normalized units of modular group rings of primary abelian groups, Serdika 6 (1980), 258-263.

14. W. Ullery, $A$ conjecture relating to the isomorphism problem for commutative group algebras, Group and Semigroup Rings, Proc. Univ. Witwatersrand Conf. (1985), North-Holland Math. Studies, No. 126, North-Holland, Amsterdam, 1986, pp. 247-252.

Department of Mathematics, University of Arizona, Tucson, Arizona 85721 